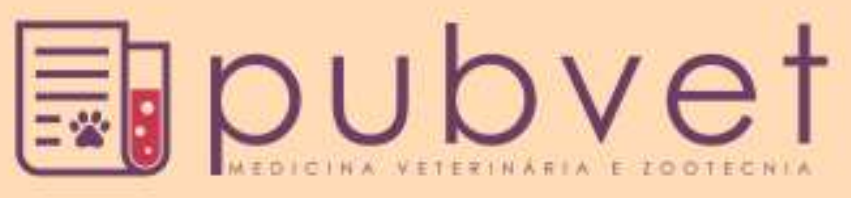

https://doi.org/10.22256/pubvet.v12n1a8.1-13

\title{
Aspectos biológicos e comportamentais de Sapajus libidinosus: Revisão
}

\author{
Ana Yasha Ferreira de La Salles ${ }^{1^{*}}$, Artur da Nóbrega Carreiro ${ }^{1 \bullet}$, Gildenor Xavier \\ Medeiros $^{2}$, José Augusto Pereira Carneiro Muniz ${ }^{36}$, Danilo José Ayres de Menezes ${ }^{1,4}$ \\ ${ }^{1}$ Universidade Federal de Campina Grande, Programa de Pós-graduação em Medicina Veterinária, Patos, Paraíba, Brasil. \\ ${ }^{2}$ Unidade Acadêmica de Medicina Veterinária, Universidade Federal de Campina Grande, Patos, Paraíba, Brasil. \\ ${ }^{3}$ Centro Nacional de Primatas (CENP), Instituto Evandro Chagas (IEC), Secretaria de Vigilância em Saúde (SVS), Ministério da Saúde (MS), \\ Ananindeua, PA, Brasil. \\ ${ }^{4}$ Universidade Federal do Rio Grande do Norte, Departamento de Morfologia, Natal, RN, Brasil. mdanayres@gmail.com \\ *Autor para correspondência, E-mail: anayasha@hotmail.com
}

\begin{abstract}
RESUMO. Os macacos-prego da espécie Sapajus libidinosus são conhecidos como os macacos mais robustos e inteligentes do Novo Mundo, e dentre as espécies neotropicais, é a que apresenta maior distribuição geográfica. Devido a isso e também pelo fácil manuseio e a facilidade de reprodução em cativeiro, o gênero vem sendo utilizado em diversas pesquisas, dada a sua proximidade filogenética com a espécie humana, servindo de modelo biológico em experimentos aplicados a essa espécie, com ampla variedade de pesquisas biomédicas. É uma espécie considerada quase ameaçada no Brasil segundo critérios de avaliação do ICMBio (2012-2014) e o estudo do comportamento animal surge como um meio de pesquisa científica que tem proporcionado importantes contribuições além do estudo do comportamento humano, neurociências, meio ambiente e bem-estar animal, também com a preservação de espécies. Considerando a relevância que a pesquisa do comportamento e biologia animal vem tomando dentro da comunidade científica, esse estudo tem por objetivo uma revisão de literatura acerca da espécie Sapajus libidinosus enfatizando, dentro do tema em questão, sua taxonomia, distribuição geográfica e população, características gerais e adaptações, vida social, alimentação e reprodução.
\end{abstract}

Palavras chave: Cebidae, comportamento animal, preservação de espécies

\section{Biological and behavioral aspects of Sapajus libidinosus: Review}

ABSTRACT. The black striped capuchin monkey of the species Sapajus libidinosus are known as the most robust and intelligent monkeys of the New World, and of the neotropical species, it is the one that presents greater geographical distribution. Due to this and also because of the easy handling and the ease of reproduction in captivity, the genus has been used in several researches, given its phylogenetic proximity to the human species, serving as a biological model in experiments applied to this species, with a wide variety of Biomedical research. It is a species considered almost threatened in Brazil according to ICMBio (2012-2014) evaluation criteria and the study of animal behavior emerges as a means of scientific research that has provided important contributions besides the study of human behavior, neurosciences, environment and animal welfare, also With the preservation of species. Considering the relevance of behavioral research and animal biology within the scientific community, this study aims to review the literature on the species Sapajus libidinosus, emphasizing, within the theme, its taxonomy, geographic distribution and population, general characteristics and adaptations, social life, food and reproduction.

Keywords: Cebidae, animal behavior, species preservation 


\title{
Aspectos biológicos y conductuales de Sapajus libidinosus: Revisión
}

\begin{abstract}
RESUMEN. Los monos de la especie Sapajus libidinosus son conocidos como los monos más robustos e inteligentes del nuevo mundo, y entre las especies neotropicales es la que presenta mayor distribución geográfica. Debido a eso y también por el fácil manejo y la facilidad de reproducción en cautiverio, el género es usado en diversas investigaciones, dada su proximidad filogenética con la especie humana, sirviendo de modelo biológico en experimentos aplicados a esa especie, con amplia variedad de investigaciones biomédicas. Es una especie considerada casi amenazada en Brasil según criterios de evaluación del ICMBio (2012-2014) y el estudio del comportamiento animal surge como un medio de investigación científica que ha proporcionado importantes contribuciones además del estudio del comportamiento humano, neurociencias, medio ambiente, bienestar animal, así como la preservación de especies. En este sentido, el estudio de la especie de Sapajus libidinosus enfatizando, dentro del tema en cuestión, su taxonomía, la distribución geográfica y la población, características generales y adaptaciones, vida social, alimentación y reproducción.
\end{abstract}

Palabras clave: Cebidae, comportamiento animal, preservación de especies

\section{Introdução}

O estudo do comportamento animal é um meio de pesquisa científica que tem proporcionado importantes contribuições para outras disciplinas, com destaque para o estudo do comportamento humano, neurociências, meio ambiente, bem-estar animal e para a educação ambiental. Quanto às espécies ameaçadas de extinção, o estudo do comportamento também surge como uma possibilidade de criação de reservas e medidas concretas de proteção.

Diante disso, os macacos-prego da espécie Sapajus libidinosus são conhecidos como os macacos mais robustos e inteligentes do Novo Mundo (Diniz, 1997). Dentre as espécies neotropicais, é a que apresenta maior distribuição geográfica (Diniz, 1997) e devido a isso e também pelo fácil manuseio e a facilidade de reprodução em cativeiro, o gênero vem sendo utilizado em diversas pesquisas.

Cada vez mais tem aumentado o número de cientistas sociais que recorrem ao comportamento animal buscando desvendar causas de problemas da sociedade humana. Os trabalhos de Harlow \& Mears (1979) sobre o desenvolvimento social de macacos Rhesus e os de Overmier \& Seligman (1967) sobre o desamparo aprendido têm tido forte influência nas teorias de desenvolvimento infantil e na psiquiatria. Uma pesquisa realizada por De Waal (2000) demonstra a cooperação e reconciliação em grupos sociais de chimpanzés. Snowdon (1999), em estudo com muriquis, macacos que não apresentam agressões entre os membros do grupo social, visa o mesmo fundamento relatado por De Waal (2000). Assim, podemos aprender como minimizar a agressão humana se compreendermos como estas espécies evitam a agressão.

Diante do exposto, e considerando a relevância que as pesquisas do comportamento e biologia animal vêm tomando dentro da comunidade científica, esse estudo tem por objetivo uma revisão de literatura sobre a espécie Sapajus libidinosus enfatizando, dentro do tema em questão, sua taxonomia, distribuição geográfica e população, características gerais e adaptações, vida social, alimentação e reprodução.

\section{Taxonomia}

Devido às várias espécies e a dificuldade de identificação das mesmas, a classificação taxonômica dos macacos-prego vem constantemente passando por uma série de revisões.

As denominações nas espécies do gênero Cebus foram criadas por Erxleben em 1777, e sofreram modificações ao longo do tempo por terem sido feitas análises mais profundas, dentre outras características, na morfologia, padrão de coloração dos pelos, dimorfismo sexual, presença ou ausência de tufos (Silva Junior, 2002). Anteriormente, de acordo com Diniz (1997), o macaco-prego era descrito como Cebus libidinosus, pertencente à subordem Anthropoidea, superfamília Platyrrhini, família Cebidae e gênero Cebus. Hershkovitz (1949) dividiu o gênero Cebus em dois grupos: os macacos-prego com tufo e sem tufo, reconhecendo como válidas quatro espécies: $C$. albifrons, $C$. olivaceus e $C$. capucinus dentre os 
macacos-prego sem tufo ou caiararas, e como única espécie de macaco-prego com tufo, o $C$. apella. Hill (1960) revisou o grupo com tufo descrevendo 16 subespécies existentes. Mittermeier et al. (1988) e Torres de Assumpção (2014) analisando o mesmo grupo também consideraram $C$. apella como única espécie válida; porém cada autor propôs cinco subespécies, classificação essa que, mesmo desatualizada, vem sendo adotada por muitos primatólogos não taxonomistas, zoológicos e Centros de Triagem de Animais Silvestres (Silva, $\underline{2010)}$.

Groves (2001) apresentou uma taxonomia alternativa para os macacos-pregos, como segue: C. apella apella Linnaeus, 1758; C. apella fatuellus Linnaeus, 1766; C. apella macrocephalus Spix, 1823; C. apella peruanus Thomas, 1901; C. apella tocantinus Lönnberg, 1939; C. apella margaritae Hollister, 1914; C. libidinosus libidinosus Spix, 1823; C. libidinosus pallidus Gray, 1866; C. libidinosus paraguayanus Fischer, 1829; C. libidinosus juruanus, Lönnberg, 1939; C. nigritus nigritus (Goldfuss, 1809); C. nigritus robustus Kuhl, $1820 ; \quad C$. nigritus cucullatus, Spix, 1823; C. xanthosternos WiedNeuwied, 1826. Silva Junior (2002) reconheceu $C$. libidinosus pallidus Gray, 1866 e C. libidinosus paraguayanus Fischer, 1829, como sinônimos de Cebus cay; e também C. libidinosus juruanus Lönnberg, 1939, como sinônimo de $C$. macrocephalus.

Em revisão mais abrangente, baseado em aspectos anatômicos de diferenças morfométricas do crânio, padrões de coloração da pelagem, silhueta do corpo e distribuição geográfica, o gênero Cebus foi subdividido em outros dois subgêneros: Cebus para os caiararas, mais esguios, distribuídos da Amazônia para o norte, e Sapajus para os macacos-prego, mais robustos e caracterizados por um topete na cabeça, espalhados da Amazônia para o sul, na mata atlântica, cerrado e caatinga (Silva Junior, 2002) e posteriormente, revisões mais recentes incluindo análises genéticas, morfológicas, comportamentais e biogeográficas, elevou cada subgênero à categoria de gênero (Silva Junior, 2002, Alfaro et al., 2012).

Silva (2010) em seus estudos adotou a divisão para os Cebus com tufo (Sapajus) contendo oito espécies, S. apella, S. macrocephalus, S. cay, $S$. nigritus, $S$. robustus, $S$. xanthosternos, $S$. flavius e S. libidinosus - espécie em estudo.

\section{Distribuição geográfica e população}

O gênero Sapajus tem a capacidade de utilizar vários ambientes, no entanto, isso não é regra geral dos primatas neotropicais. Quando se observa um mapa de distribuição geográfica de determinada espécie, tem-se a impressão de que ela distribui-se uniformemente e será encontrada em qualquer local dentro desta área. Todavia, logicamente isto não ocorre, e vai ser dependente, na maioria das vezes, do tipo de vegetação, clima, solo, topografia, disponibilidade de alimento, abrigo, ou qualquer outra necessidade (Auricchio, 1995).

De hábitos alimentares generalistas e com grande flexibilidade comportamental e ecológica, o gênero Sapajus habita quase toda a região neotropical. O seu habitat é o mais diversificado dos primatas neotropicais, utilizando todos os estratos arbóreos de florestas chuvosas inundáveis ou não, florestas ripárias e formações arbóreas e arbustivas dos biomas Cerrado e Caatinga, palmeiras, campos e manguezais, não sendo restrito a habitat primários, podendo ocupar áreas altamente fragmentadas e perturbadas (Auricchio, 1995, Rylands \& Mittermeier, 2009).

A extensão de ocorrência da espécie é maior que $20.000 \mathrm{~km}^{2} \mathrm{e}$ infere-se que a área de ocupação seja maior que $2.000 \mathrm{~km}^{2}$, conforme critérios IUCN. Apresentam densidade de 35 indivíduos por $\mathrm{km}^{2}$ e biomassa de $96 \mathrm{~kg}$ por $\mathrm{km}^{2}$. O tamanho da população total remanescente não é conhecido, mas estima-se que o número de indivíduos maduros deste gênero seja superior a 10.000 (Auricchio, 1995).

Sapajus libidinosus é endêmico ao Brasil, ocorrendo nos estados de Alagoas, Bahia, Ceará, Goiás, Maranhão, Minas Gerais, São Paulo, Piauí, Rio Grande do Norte, Tocantins, Pernambuco e Paraíba, onde é residente e nativo. Ocorre no nordeste do Brasil, na parte oriental do estado do Maranhão, a partir da bacia dos rios Mearim e Itapecuru, atravessando os estados do Piauí, Ceará, Rio Grande do Norte, Paraíba, Pernambuco e Alagoas. A distribuição segue para oeste do rio São Francisco pelo Cerrado. Em Tocantins, Goiás, oeste de Minas Gerais e norte de São Paulo, parte do oeste da Bahia e talvez nordeste de Mato Grosso, sendo substituído por Sapajus apella ao norte, na transição para a Floresta Amazônica e Florestas do Mato Grosso (Silva Junior, 2002), e por Sapajus cay a oeste. O mesmo autor indica que o limite ocidental se encontra a leste do rio Araguaia e o limite sul fica entre a região do Rio 
Grande no oeste de Minas Gerais (Triângulo Mineiro) e extremo norte de São Paulo.

No gênero Sapajus, o tamanho do grupo pode variar de dois a mais de 50 indivíduos, mas, no geral, os grupos são compostos de 6 a 30 indivíduos (Freese \& Oppenheimer, 1981). De acordo com Robinson \& Janson (1987), os grupos de Sapajus podem ter de 10 a 35 indivíduos; porém as espécies de Cebus - macacos-prego "sem tufos"-, formam grupos maiores. Foi registrado densidade de 3 grupos $/ \mathrm{km}^{2}$ e 6,7 grupos $/ \mathrm{km}^{2}$ no Parque Estadual Altamiro de Moura Pacheco, Goiânia, GO (Villar, 2006). Na Serra da Capivara a densidade da espécie variou entre 7,1 e 11,3 indivíduos $/ \mathrm{km}^{2}$, a depender do método de análise (Moura \& Lee, 2004).

A tendência populacional encontra-se em declínio, baseando-se na perda ocorrida e contínua de hábitat. A Caatinga, por exemplo, teve mais de $50 \%$ de sua área desmatada até 2009 segundo informações do IBGE e, no Cerrado, a perda é estimada em cerca de 50\% (Machado et al., 2004). Além da alta pressão de caça, são vítimas comuns do comércio ilegal de animais de estimação. Porém, devido a plasticidade da espécie, o declínio populacional não deve ser considerado na mesma proporção da perda e fragmentação do habitat, mas supõe-se que este deve se aproximar de $30 \%$ da população.

\section{Características gerais e adaptações}

Os primatas neotropicais são mamíferos placentários exclusivamente arborícolas. A escolha por este ambiente adaptou-os a uma série de peculiaridades da Ordem como, por exemplo, a visão tornar-se o sentido mais desenvolvido. $\mathrm{O}$ olfato, no ambiente arbóreo, não era eficiente o bastante para seguir rastros ou procurar alimentos, sendo utilizado principalmente no reconhecimento entre indivíduos (Auricchio, 1995).

Pesquisas relacionadas ao estudo da visão em primatas neotropicais têm ganhado espaço no meio científico (Altavini, 2012, Saletti, 2010, Silva, 2012, Henriques et al., 2014, Belham, 2013). Diferentemente dos primatas do Velho Mundo que possuem tricromacia uniforme, os primatas do Novo Mundo apresentam um polimorfismo da visão de cores relacionado ao sexo, no qual todos os machos são dicromatas e as fêmeas podem ser dicromatas ou tricromatas, com duas exceções: o monocromatismo do gênero Aotus e o tricromatismo em ambos os sexos no gênero Alouatta. Estudos sugerem que a visão de cores facilita a identificação e procura de alimentos e potenciais predadores (Jacobs et al., 1993, Jacobs et al., 1996, Jacobs, 2007, Araújo Júnior et al., 2008). Além disso, esta visão com órbitas totalmente circundadas por ossatura permitiu uma boa noção de distância, imprescindível à locomoção no ambiente arbóreo.

Supõe-se que o volume corpóreo é o mais forte indicador das adaptações fisiológicas, ecológicas e comportamentais que um animal pode apresentar. A grande variação deste quesito dentre as espécies é proveniente de várias modificações que ocorreram como forma de adaptação às novas condições ambientais que apareciam (Auricchio, 1995). Segundo Rowe (1996), animais do gênero Sapajus possuem um tamanho de corpo médio, dentre os primatas neotropicais, e apresentam peso entre 1,4 e $4,8 \mathrm{~kg}$. O comprimento total da cabeça e corpo varia de 350 e $488 \mathrm{~mm}$ e o da cauda, de 375 a $554 \mathrm{~mm}$. Poucas são as espécies que apresentam dimorfismo sexual acentuado, sendo na maioria caracterizado pela genitália, volume corpóreo, pequenas variações de comportamento e por secreção de feromônios. Nos adultos do gênero Sapajus, observa-se que os machos são maiores que as fêmeas em todas as espécies ( $\underline{\text { Silva }}$ Junior, 2002). Além disso, os pêlos da face, formato da cabeça e as proporções corporais são frequentemente diferenciações entre os sexos (Freese \& Oppenheimer, 1981).

Uma das adaptações mais notáveis nos primatas neotropicais é a capacidade de preensibilidade da cauda encontrada nos gêneros de maior porte, como no Ateles e Brachyteles. Nesses animais a região inferior da ponta da cauda apresenta uma região glabra, formando uma "palma" e permitindo sua utilização como um quinto membro, aumentando sua mobilidade e velocidade, e muito útil devido a capacidade de braquiação desses animais. O gênero Sapajus apresenta cauda semi-prêensil, menos desenvolvida que a dos anteriores, sem palma subcaudal, porém lhes conferindo uma enorme agilidade e ainda utilizada, durante o forrageio, tanto para a suspensão como para o apoio, sendo capaz de suportar o peso de um adulto apenas por curtos períodos (Fragaszy \& Bard, 1997).

Os Sapajus libidinosus possuem coloração que tende a um amarelo ou bege, com os membros de cor escura com proximidade ao preto, assim como o topete, que possui forma espessa. Eles possuem braços quase tão longos quanto as pernas, polegares oponíveis e grande destreza (Robinson 
\& Janson, 1987). Evolutivamente, os primatas neotropicais trocaram suas unhas em forma de garras (falculae) por unhas planas (ungulae) que estão relacionadas a uma maior sensibilidade, com exceção para os Callitrichidae, que retornaram às unhas em forma de garra devido seu modo de vida, para facilitar a obtenção de alimentos (Auricchio, 1995).

As mandíbulas são robustas e os dentes grandes e compactos, bem adaptados ao seu forrageio extrativo. Suas mãos são muito manipulativas e ágeis, sendo os únicos macacos do Novo Mundo capazes de utilizar ferramentas na natureza a fim de facilitar a exploração de recursos (Rocha et al., 1998, Fragaszy \& Bard, 1997, Moura \& Lee, 2004). Vários trabalhos vêm sendo realizados quanto a essa peculiaridade da espécie em estudo, expondo métodos de resolução de problemas (Silva, 2008, Cardoso, 2013), efeitos do uso de ferramentas no comportamento e bemestar desses animais (Camargo, 2012, Cutrim, 2013); otimização e observação de uso desses materiais em animais de cativeiro e vida livre (Emidio \& Ferreira , 2012, Falótico et al, 2017; Lessa et al., 2011), e utilização desses métodos de manipulação na brincadeira social dentro do grupo (Carvalho, 2013).

Esse primata neotropical tem atraído à atenção devido ao seu índice de encefalização, que é maior do que a dos primatas do Velho Mundo, como o gorila (Gorilla gorilla) e o chimpanzé (Pan troglodytes), possuindo o maior tamanho relativo do cérebro em relação ao seu tamanho de corpo dentre os macacos do Novo Mundo (Fragaszy \& Bard, 1997, Garber \& Lavallee, 1999) e o segundo maior índice de capacidade craniana dentre os primatas (Martin, 1990), apresentando ainda comportamento cultural complexo e memória bem desenvolvida, sendo talvez o primata mais inteligente das Américas (Lopes, 2004). Além disso, podem chegar a viver, em cativeiro, 47 anos, o que é um tempo de vida maior que o de outros primatas de mesmo tamanho (Fedigan et al., 1996).

Dada a sua proximidade filogenética, primatas como os macacos-prego vêm sendo utilizados como modelo biológico em experimentos aplicados à espécie humana, com ampla variedade de pesquisas biomédicas e comportamentais, prevenção e cura de doenças, controle de produtos farmacêuticos, produção e desenvolvimento de vacinas, sendo ainda modelos úteis para estudos de cirurgias experimentais como transplante de órgãos, neurociência e doenças cardiovasculares (Popilskis et al., 2008).

\section{Vida social}

Os primatas, devido suas atribuições físicas e mentais, possuem também variações de estrutura social que são respostas evolutivas ao meio ambiente.

Os grupos do gênero Sapajus apresentam, em geral, apenas um ou dois machos adultos, com quantidade maior de fêmeas adultas em relação à quantidade de machos adultos, sendo, portanto, classificados como poligínicos, apesar de que em cativeiro adotam postura poligâmica. Os machos, quando atingem a maturidade sexual, migram de seu grupo natal, enquanto as fêmeas são filopátricas, ou seja, se mantêm no grupo de origem (Bicca-Marques et al., 2006).

Nem sempre apresentam uma hierarquia de dominância, mas, no geral, o macho dominante e a fêmea dominante podem ser facilmente identificados. Em Cebus e Sapajus interações entre machos de grupos sociais distintos são comumente agressivas e em Cebus a entrada de um macho adulto num grupo social geralmente está associada a altos níveis de agressão, principalmente, quando o macho imigrante tentar apoderar-se da posição de dominância do macho alfa (Jack \& Fedigan, 2004).

Quanto ao comportamento observado nesses primatas neotropicais, o de catação é visto como a interação social mais comum (Sussman et al., 2005) e consiste de uma inspeção realizada no pelo de outro indivíduo, removendo sujeira e ectoparasitas com as mãos, língua e dentes (Schino, 2001). Há duas possibilidades de hipóteses sobre a função da catação: uma determina que tenha como função a higiene do pelo (Hutchins \& Barash, 1976); enquanto a outra afirma que representa uma função social, como forma de estabelecer e manter relações dentro do grupo (Stammbach \& Kummer, 1982), assim como reduzir as tensões sociais (Boccia, 1987, Schino et al., 1988). Di Bitetti (1997) verificou que os pares de indivíduos que realizam catação com maior frequência são os mesmos que formam alianças mais fortes. Alguns estudos realizados com Cebus capucinus relataram que as fêmeas dominantes recebem a maior parte das catações (Perry et al., 2017, Rose, 1998). Entretanto, um estudo realizado Cebus apella em cativeiro indicou que as fêmeas dominantes realizam 
catações com maior frequência nas fêmeas subordinadas do que o inverso (Parr et al., 1997).

A marcação por cheiro é outro comportamento amplamente utilizado pelos primatas neotropicais. As secreções glandulares - circungenitais, suprapúbicas e do esterno são passadas nas árvores para marcar território, protegê-lo da competição, e agem na atração sexual, no reconhecimento individual, indicam o status social, ameaça, e auxiliam no reconhecimento mãe-filhote (Auricchio, 1995).

Outro tipo de comportamento é o de vocalização, sendo bastante característico em alguns gêneros de primatas neotropicais, como é o caso do Callicebus e dos Alouattas, que apresentam o osso hioide mais desenvolvido, promovendo um ronco que é audível à longa distância. Esse tipo de comunicação é importante por diminuir a ocorrência de brigas, definir territórios e facilitar a comunicação entre animais a certa distância separada pela vegetação (Auricchio, 1995). O uso da vocalização relacionada a comportamentos específicos como proximidade, interação social, conduta alimentar, comportamento sexual, encontro entre grupos, entre outros (Gros-Louis et al., 2008, Leliveld et al., 2011) tem sido registrado para diferentes espécies do gênero Cebus e Sapajus (Boinski, 1993, Boinski \& Campbell, 1996, Di Bitetti \& Janson, 2001, Di Bitetti, 2003).

Estudos vêm sendo desenvolvidos quanto a esse tipo de comportamento, relatando observações de vocalizações quanto à ameaça e perseguição em Sapajus libidinosus (Pinha, 2007), episódios de vocalizações de alimentação na mesma espécie (Ferreira, 2016) e avaliação da comunicação vocal de Sapajus flavius em vida livre (Araújo, 2013).

Os inimigos naturais da espécie Sapajus libidinosus são a harpia, o gavião pega-macacos, a onça e a irara. Podem ser observadas associações com outras espécies de primatas e também com aves como Accipiter bicolor que se aproveita de insetos dispersados por eles em seus forrageios (Auricchio, 1995). Podem também exercer um importante papel na propagação e transmissão de patógenos para os animais domésticos e para o homem, à proporção que estes invadem seus habitats ou estabelecem contato com os mesmos, ainda que no meio urbano (Fragaszy \& Bard, 1997).

\section{Alimentação}

Durante a infância, os mamíferos obtêm sua nutrição por meio do leite materno, e à medida que vai se desenvolvendo e tornam-se capazes de obter e digerir outros tipos de alimento passa a depender cada vez menos dessa fonte de alimento (Lee, 1996). A partir do desmame, os jovens já se tornam responsáveis pela obtenção de seu próprio alimento, no entanto, isto pode representar redução na energia obtida. Fragaszy \& Bard (1997) encontraram uma desaceleração no crescimento após desmame em Sapajus sugestivo de redução na obtenção de nutrientes.

O comportamento de forrageio inclui a localização, obtenção, processamento e ingestão de alimentos. As habilidades manipulativas necessárias para o forrageamento de primatas precisam ser adquiridas ao longo da juventude (Joffe, 1997). Agostini \& Visalberghi (2005) afirmam que a eficiência dos juvenis no forrageamento é menor que a dos adultos. Da mesma forma, Janson \& Van Schaik (1993) complementam essa afirmativa relatando que a menor competência dos juvenis aplica-se ao menor tamanho e força, mas em grande parte é direcionada à falta de experiência que pode ser melhorada por meio de observação, tentativa e erro ou prática. No estudo de Resende (2004), sobre a ontogenia de comportamentos manipulativos de macacos-prego, os animais só conseguiram obter sucesso no uso de ferramentas para quebra de cocos após os dois anos de idade, e ainda em taxas bastante inferiores às dos adultos. Devido a sua grande capacidade de manipulação, e exploração de recursos, o Sapajus libidinosus é considerada uma espécie oportunista generalista, apresentando dieta bastante variável, inclusive incluindo itens que não são utilizados por outros primatas (Visalberghi, 1988). Apresentam sua dieta baseada principalmente em frutos $(60 \%)$, mas também incluem sementes, castanhas, flores, gomas, néctar, fungos, seiva, ovos, insetos, aracnídeos, pequenos vertebrados e até algumas espécies de ostras e caranguejos encontrados em regiões de manguezal. Apresentam grande ingestão e, devido à grande capacidade de manipulação desses frutos e sementes, esses animais se tornam também muito importantes no processo de disseminação destas, contribuindo para a estabilidade do ecossistema (Rocha, 1992).

A importância de invertebrados na alimentação de Sapajus libidinosus é um aspecto bastante discutido por alguns pesquisadores 
(Oppenheimer, 1968, Spironelo, 1991, Guillotin \& Dubost, 1994) que descrevem frutos como a dieta básica dos macacos-prego (Spironelo, 1991) e (Galetti \& Pedroni, 1994), sendo a primeira uma importante opção em épocas de seca e em ambientes que sofreram grande degradação e perturbação.

Por serem primatas de porte médio e apresentarem esta capacidade de manipulação, estes animais exploram mais facilmente alimentos mais pesados e com proteção como sementes e nozes. Segundo Spironelo (1991), os frutos são utilizados em maior proporção na estação chuvosa. Em períodos de escassez de alimentos, frutos de Astrocaryum sp. (Palmae) são consumidos (Terborgh, 1983). Portanto outros recursos já citados anteriormente surgem como importante suplemento alimentar para Sapajus libidinosus em épocas de restrições de alimentos, considerando também o ambiente habitado pelo grupo social, visto que apresenta o habitat mais diversificado dos primatas neotropicais, utilizando todos os estratos arbóreos.

Simpson \& Raubenheimer (2012) relatam em seu estudo que não se pode responder a praticamente nenhum questionamento sobre comportamento ou evolução de primatas - ou inclusive de outras classes de animais, sem que antes estejam claras as características de sua dieta. Todos os aspectos da biologia animal apresentam relação à nutrição, visto que os nutrientes e a energia obtida pela alimentação servem como combustível para o funcionamento do organismo (Raubenheimer, 2010). Assim, sendo os principais determinantes da sobrevivência e reprodução, crescimento populacional, padrões de atividade e ciclos de vida dos seres vivos.

Dessa forma, a dieta passou a ocupar um espaço importante na pesquisa com primatas. Em especial à espécie em estudo, gerou pesquisas relacionadas aos parâmetros nutricionais associados à dieta em ambiente de caatinga e cerrado (Santos, 2015), memória relacional espacial e não espacial associada ao forrageamento de animais em cativeiro (Waga, 2008), comportamento posicional e uso de substratos (Biondi, 2010), efeito de novidade alimentar em grupo em semi liberdade (Cardoso, 2008), hierarquia de dominância durante a obtenção de alimento em cativeiro (Collaço, 2008), organização sequencial e otimização do comportamento na quebra de frutos encapsulados (Corat, 2016), influência da disponibilidade de alimentos sobre o comportamento (Sacramento, 2014).

\section{Reprodução}

O comportamento reprodutivo nas diferentes espécies de animais pode sofrer influência de vários fatores, tais como: oferta de recursos alimentares, mudanças na cor da genitália e, principalmente, pelos níveis dos hormônios sexuais (Carlson, 2002).

Em muitas espécies de primatas, características mudanças hormonais e morfológicas indicam amadurecimento reprodutivo. $\mathrm{O}$ amadurecimento reprodutivo ou a puberdade observados na mulher e em algumas espécies de primatas são caracterizados pelo desenvolvimento das estruturas do organismo, iniciando com $\mathrm{o}$ aparecimento da primeira menstruação (Carnegie et al., 2005). Em Cebus spp. e Sapajus spp. o ciclo menstrual, quando comparado ao dos primatas do velho mundo, correlacionam-se em parâmetros morfológicos e endócrinos (Ortiz et al., 2005) e em Sapajus libidinosus o ciclo reprodutivo geralmente não recomeça até que a prole da gestação anterior se desenvolva (Rodrigues, 2010).

No gênero Sapajus o ciclo menstrual dura entre 15 a 21 dias, apresentando um sangramento bem discreto. As fêmeas atingem a maturidade sexual entre três e quatro anos de idade. No entanto, dificilmente reproduzem até que atinjam inteiramente o seu peso adulto que ocorre em torno dos 5 anos de idade (Fragaszy \& Bard, 1997). Os machos podem demorar até o sétimo ou oitavo ano de vida para atingir maturidade sexual, no entanto expressam o seu interesse pelas fêmeas por volta dos três anos de idade, enquanto os juvenis se envolvem em comportamentos e brincadeiras sexuais. Em cativeiro, a maturidade pode ocorrer de forma mais precoce (Ortiz et al., 2005), e animais desse gênero podem reproduzir até aproximadamente os 25 anos nesse ambiente (Auricchio, 1995, Bicca-Marques et al., 2006).

De acordo com Carnegie et al. (2005) em estudo com Cebus capucinus na fase periovulatória foi observado que, embora as fêmeas não ofereçam pistas morfológicas ou comportamentais de que a ovulação esteja ocorrendo, os machos têm a capacidade de detectá-la. Complementando tal estudo, Rodrigues (2010) relata que o melhor indicador de detecção da fase do ciclo se dá pelo aumento na demonstração de comportamentos sexuais do 
macho dirigidos às fêmeas no período periovulatório. Carosi \& Visalberghi (2002) e Carosi et al. (2005) já descrevem que durante o período fértil, as fêmeas solicitam ativamente o macho dominante pelas perseguições, exibições faciais, vocais e posturais (Carosi \& Visalberghi, 2002, Carosi et al., 2005).

Primatas do gênero Sapajus possuem um comportamento de cuidado com a prole que surgiu do fato de serem constituídos por animais que vivem em grupos, sem construção de ninhos. Isto fez com que tivessem a responsabilidade de carregarem suas crias, aproximando mais os pais de seus filhotes (Bicca-Marques et al., 2006). A gestação nesse gênero dura em média cinco a seis meses, gerando apenas um filhote, com cerca de $260 \mathrm{~g}$, que é carregado pelos pais e outros indivíduos do grupo até o desmame que ocorre aos 8 meses, aproximadamente (Auricchio, 1995, Bicca-Marques et al., 2006).

Existem dois modelos básicos de reprodução considerados entre os primatas: o de fluxo contínuo, no qual os nascimentos ocorrem dispersos durante todo o ano, e o de picos, com os nascimentos ocorrendo na mesma época do ano, todos os anos. No entanto, nenhuma espécie segue estes padrões de forma rigorosa, principalmente em primatas, com a maioria das espécies apresentando maior incidência de nascimentos no início da época de maior disponibilidade de alimentos (Auricchio, 1995). Bicca-Marques et al. (2006) afirmam que alguns estudos na natureza e em cativeiro mostram que Sapajus libidinosus reproduz sazonalmente ou apresenta picos de nascimentos entre os meses de outubro a fevereiro. Este período que coincide com a época de maior disponibilidade de frutos e insetos. No entanto, Auricchio (1995) relata que animais do gênero Sapajus não apresentam estação reprodutiva.

No que se refere aos primatas neotropicais, estudos a respeito do comportamento reprodutivo (Carosi \& Visalberghi, 2002, Fedigan et al., 2008, Fedigan \& Jack, 2011), anatomia do sistema reprodutor (Veras et al., 2007, Pissinatti et al., 2008, Branco et al., 2010), entre outros aspectos ligados à biologia reprodutiva, tem sido realizados. No entanto, poucos são os estudos relativos à reprodução que abordam especificamente a espécie Sapajus libidinosus, se resumindo à relação de aplicação hormonal com o status hierárquico e a reprodução de machos adultos (Edaes, 2012); ciclo reprodutivo de animais criados em cativeiro (Rodrigues, 2010) e relação entre a aplicação de hormônios e o ciclo menstrual de fêmeas cativas (Rodrigues, 2016).

\section{Conclusão}

A espécie Sapajus libidinosus é largamente distribuída nas regiões Norte, Nordeste e Sudeste do Brasil, e apesar disso, poucos são os relatos encontrados na literatura científica que enriqueçam o conhecimento sobre ela. $\mathrm{O}$ estudo do comportamento animal vem proporcionando importantes contribuições, desde o estudo do comportamento humano até na preservação de espécies ameaçadas de extinção, como uma possibilidade de criação de reservas e medidas de proteção. Diante disso, estudos que envolvam espécies ameaçadas é de extrema importância para os estudantes e pesquisadores da área.

\section{Referências Bibliográficas}

Agostini, I. \& Visalberghi, E. 2005. Social influences on the acquisition of sex-typical foraging patterns by juveniles in a group of wild tufted capuchin monkeys (Cebus nigritus). American Journal of Primatology, 65, 335-351.

Alfaro, J. W. L., Ssilva, J. S. \& Rylands, A. B. 2012. How different are robust and gracile capuchin monkeys? An argument for the use of Sapajus and Cebus. American Journal of Primatology, 74, 273-286.

Altavini, T. S., Henriques, L. D., Bonci, D. M. O., Nagy, B V., Ventura, D. F. \& Pessoa, V. F. 2012. Using the Hard, Randy, and Rittler Test to Evaluate Color Vision in Capuchins (Cebus libidinosus). International Journal of Primatology, 33, 1467-1476

Altavini, T. S. 2009. Avaliação da percepção de cores em macaco-prego (Cebus libidinosus) pelo teste pseudoisocromático de HRR (Hard, Rand e Rittler). Dissertação (Mestrado), Instituto de Biologia, Universidade de Brasília, Brasília. 58f.

Araújo, M. B. 2013. Comunicação vocal em Sapajus flavius na natureza Recife. Dissertação (mestrado), Pós-graduação em Biologia Animal, Universidade Federal de Pernambuco, Recife. $90 \mathrm{f}$.

Araújo Júnior, A. C., Didonet, J. J., Araújo, C. S., Saletti, P. G., Borges, T. R. J. \& Pessoa, V. F. 2008. Color vision in the black howler monkey (Alouatta caraya). Visual Neuroscience, 25, 243-248. 
Auricchio, P. 1995. Primatas do Brasil. Terra Brasilis Editora Ltda., Arujá, São Paulo, Brasil. Terra Brasilis Editora Ltda, São Paulo, Brasil.

Belham, F. S. 2013. Influência da valência emocional de estímulos na memória operacional visuo-espacial de humanos e macacos-prego (Cebus libidinosus). Dissertação (Mestrado), Programa de pósgraduação em Biologia animal, Universidade de Brasília, Brasília. 123f.

Bicca-Marques, J. C., Silva, V. M. \& Gomes, D. F. 2006. Ordem Primates. In: Reis, N. R., Peracchi, A. L., Pedro, W. A. \& Lima, I. P. (eds.) Mamíferos do Brasil. Londrina, Paraná.

Biondi, L. C. M. 2010. Comportamento posicional e uso de substrato de macacos-prego Cebus Libidinosus Spix, 1823. Dissertação (Mestrado), Programa de Pós-Graduação em Psicologia, Instituto de Psicologia da Universidade de São Paulo, São Paulo. 120 f.

Boccia, M. L. 1987. The physiology of groomingA test of the tension reduction hypothesis. American Journal of Primatology, 4, 399-418.

Boinski, S. 1993. Vocal coordination of troop movement among white-faced capuchin monkeys, Cebus capucinus. American Journal of Primatology, 30, 85-100.

Boinski, S. \& Campbell, A. F. 1996. The huh vocalization of White-faced Capuchins: a spacing call disguised as a food call? Ethology, 102, 826-840.

Branco, E., Lacreta Jr, A. C. C., Ishizaki, M. N., Pereira, W. L. A., Meneses, A. M. C., Muniz, J. A. P. C. \& Fioretto, E. T. 2010. Morfologia macroscópica e morfometria do aparelho urogenital do macaco de cheiro (Saimiri sciureus Linnaeus, 1758). Biotemas, 23, 197202.

Camargo, M. R. 2012. O efeito do uso de ferramentas no comportamento e no bem-estar de macacos-prego (Sapajus libidinosus) cativos. Dissertação (Mestrado), Programa de Pós-Graduação em Ciências do Comportamento, Instituto de Psicologia da Universidade de Brasília, Brasília. 62f.

Cardoso, R. M. 2008. O efeito da novidade alimentar e comportamentos associados ao forrageio em um grupo semi-livre de macacosprego (Cebus libidinosus). Dissertação (Mestrado), Departamento de Psicologia, Pontífica Universidade Católica de Goiás. Goiania. 59f.
Cardoso, R. M. 2013. Resolução de problema por macacos-prego selvagens (Sapajus libidinosus) de duas populações com diferentes repertórios de uso de ferramentas. Tese (Doutorado) Programa de Pós-Graduação em Psicologia, Instituto de Psicologia da Universidade de São Paulo, São Paulo. 162 f.

Carlson, N. R. 2002. Fisiologia do Comportamento. Editora Manole, São Paulo, Brasil.

Carnegie, S. D., Fedigan, L. M. \& Ziegler, T. E. 2005. Behavioral indicators of ovarian phase in white-faced capuchins (Cebus capucinus). American Journal of Primatology, 67, 51-68.

Carosi, M., Linn, G. S. \& Visalberghi, E. 2005. The sexual behavior and breeding system of tufted capuchin monkeys (Cebus apella). Advances in the Study of Behavior, 35, 105149.

Carosi, M. \& Visalberghi, E. 2002. Analysis of tufted capuchin (Cebus apella) courtship and sexual behavior repertoire: changes throughout the female cycle and female interindividual differences. American Journal of Physical Anthropology, 118, 11-24.

Carvalho, C. E. 2013. Estudo da motivação para manipulação e para brincadeira social em macacos-prego (Sapajus sp.). Dissertação (Mestrado), Instituto de psicologia, Universidade de São Paulo, São Paulo. 94f.

Collaço, B. J. R. 2008. Hierarquia de dominância durante a obtenção de alimento em Cebus Apella e Cebus Libidinosus. Dissertação (Mestrado), Programa de Pós-Graduação em Psicobiologia, Universidade Federal do Rio Grande do Norte, Natal. 57 f.

Corat, C. S., Siqueira, S. \& Ottoni, E. B. 2016. Sequential organization and optimization of the nut-cracking behavior of semi-free tufted capuchin monkeys (Sapajus sp.). Primates, 57, 113-121

Cutrim, F. H. R. 2013. Padrão comportamental e uso de ferramentas em macacos-prego (Sapajus libidinosus) residentes em manguezal. Tese (Doutorado), Programa de pós-graduação em psicologia, Universidade de São Paulo, São Paulo. 114f.

De Waal, F. B. M. 2000. Attitudinal reciprocity in food sharing among brown capuchin monkeys. Animal Behaviour, 60, 253-261. 
Di Bitetti, M. S. 1997. Evidence for an important social role of allogrooming in a platyrrhine primate. Animal Behaviour, 54, 199-211.

Di Bitetti, M. S. 2003. Food-associated calls of tufted capuchin monkeys (Cebus apella nigritus) are functionally referential signals. Behaviour, 140, 565-592.

Di Bitetti, M. S. \& Janson, C. H. 2001. Social foraging and the finder's share in capuchin monkeys, Cebus apella. Animal Behaviour, 62, 47-56.

Diniz, L. S. M. 1997. Características $e$ distribuição geográfica. Primatas em cativeiro manejo e problemas veterinários enfoque para espécies neotropicais. Ícone, São Paulo.

Edaes, M. M. L. 2012. Relação entre testosterona, status hierárquico e reprodução em machos adultos de macacos-prego (Sapajus libidinosus). Relatório Final de Iniciação Científica, Departamento de Psicologia experimental, Universidade de São Paulo, São Paulo. 26f.

Emidio, R. A. \& Ferreira, R. G. 2012. Energetic Payoff of Tool Use for Capuchin Monkeys in the Caatinga: Variation by Season and Habitat Type. American Journal of Primatology, 74, 332-343

Falótico, T., Luncz, L. V., Svensson, M. S. \& Haslam, M. 2017. Cashew Nut Positioning during Stone Tool Use by Wild Bearded Capuchin Monkeys (Sapajus libidinosus). Folia Primatologica, 21, 8

Fedigan, L. M., Carnegie, S. D. \& Jack, K. M. 2008. Predictors of reproductive success in female white-faced capuchins (Cebus capucinus). American Journal of Physical Anthropology, 137, 82-90.

Fedigan, L. M. \& Jack, K. M. 2011. Two girls for every boy: the effects of group size and composition on the reproductive success of male and female white-faced capuchins. American Journal of Physical Anthropology, 144, 317-326.

Fedigan, L. M., Rosenberger, A. L., Boinski, S., Norconk, M. A. \& Garber, P. A. 1996. Critical issues in Cebine evolution and behavior. Plenum Press, New York.

Ferreira, L. G. 2016. Vocalizações de alimentação de macacos-prego (Sapajus libidinosus): investigação sobre fatores causais e função. Dissertação (Mestrado), Programa de Pós-
Graduação em Psicologia, Universidade de São Paulo, São Paulo. 76 f.

Ferreira, R. G., Jerusalinsky, L., Silva, T. C. F., Fialho, M. S., Roque, A. A.; Fernandes, A. \& Arruda, F. 2009. On the occurrence of Cebus flavius (Schreber 1774) in the Caatinga, and the use of semi-arid environments by Cebus species in the Brazilian state of Rio Grande do Norte. Primates, 50, 357-362.

Fragaszy, D. M. \& Bard, K. 1997. Comparison of development and life history in Pan and Cebus. International Journal of Primatology, 18, 683701.

Freese, C. \& Oppenheimer, J. R. 1981. The capuchin monkeys, genus Cebus. In: CoimbraFilho, A. F. \& A., M. R. (eds.) Ecology and behavior of neotropical primates. Academia Brasileira de Ciências, Rio de Janeiro, Brasil.

Galetti, M. \& Pedroni, F. 1994. Seasonal diet of capuchin monkeys (Cebus apella) in a semideciduous forest in south-east Brazil. Journal of Tropical Ecology, 10, 27-39.

Garber, P. A. \& Lavallee, A. 1999. Experimental approaches to the study of primate cognition in natural and near-to-wild field settings. In: Garber, P. A. \& Leigh, S. (eds.) Readings in the biological bases of human behavior. Needham Heights: Pearson Custom. Pearson Custom, Needham Heights, EUA.

Gros-Louis, J. J., Perry, S. E., Fichtel, C., Wikberg, E., Gilkenson, H., Wofsy, S. \& Fuentes, A. 2008. Vocal repertoire of Cebus capucinus: acoustic structure, context, and usage. International Journal of Primatology, 29, 641-670.

Groves, C. 2001. Primate taxonomy. Smithsonian series in comparative evolutionary biology. Smithsonian, Washington.

Guillotin, M. \& Dubost, G. 1994. Food Choice and Food Competition Among the Three Major Primate Species of French Guiana. Journal of Zoology, 233, 551-579.

Harlow, H. F. \& Mears, C. 1979. The human model: Primate perspectives. Vh Winston, Wasshingtown.

Henriques, L. D., Goulart, P. R., Oliveira, J. C., Bonci, D. M. O., Souza, G. S., Silveira, L. C. L., Galvão, O. F. \& Ventura, D. F. 2014. Opsin gene identification and evaluation of color vision in an albino capuchin monkey (Sapajus/Cebus sp). Investigative Ophthalmology \& Visual Science, .55, 268 
Hershkovitz, P. 1949. Mammals of northern Colombia. Preliminary report No. 4: Monkeys (Primates), with taxonomic revisions of some forms. Proceding of the United States National Museum, 98, 323-427.

Hill, W. C. O. 1960. Primates Comparative Anatomy and Taxonomy IV Cebidae. Edinburgh University Press.

Hutchins, M. \& Barash, D. P. 1976. Grooming in primates: implications for its utilitarian function. Primates, 17, 145-150.

Jack, K. M. \& Fedigan, L. 2004. Male dispersal patterns in white-faced capuchins, Cebus capucinus: Part 2: patterns and causes of secondary dispersal. Animal Behaviour, 67, 771-782.

Jacobs, G. H. 2007. New World monkeys and color. International Journal of Primatology, 28, 729-759.

Jacobs, G. H., Deegan, J. F., Neitz, J., Crognale, M. A. \& Neitz, M. 1993. Photopigments and color vision in the nocturnal monkey, Aotus. Vision Research, 33, 1773-1783.

Jacobs, G. H., Neitz, M., Deegan, J. F. \& Neitz, J. 1996. Trichromatic colour vision in New World monkeys. Nature, 382, 156-158.

Janson, C. H. \& Van Schaik, C. P. 1993. Ecological risk aversion in juvenile primates: slow and steady wins the race. Oxford University Press, Nova York, EUA.

Joffe, T. H. 1997. Social pressures have selected for an extended juvenile period in primates. Journal of Human Evolution, 32, 593-605.

Lee, P. C. 1996. The meanings of weaning: growth, lactation, and life history. Evolutionary Anthropology Issues News and Reviews, 5, 87-98.

Leliveld, L. M. C., Scheumann, M. \& Zimmermann, E. 2011. Acoustic correlates of individuality in the vocal repertoire of a nocturnal primate (Microcebus murinus). The Journal of the Acoustical Society of America, 129, 2278-2288.

Lessa, M. A. M., Galvão, O. F. \& Delage, P. E. G. A. 2011. Um Caso de Uso Espontâneo de Ferramenta por um Macaco-Prego (Cebus apella) Mantido em Cativeiro. Neotropical Primates, 18, 44-49.

Lopes, R.J. 2004. Gênio da Selva. Scientific American Brasil, São Paulo, Brasil.

Machado, R. B., Ramos Neto, M. B., Pereira, P. G. P., Caldas, E. F., Gonçalves, D. A., Santos,
N. S., Tabor, K. \& Steininger, M. 2004. Estimativas de perda da área do Cerrado brasileiro. Conservation International do Brasil. Brasília, Brasil.

Martin, R. D. 1990. Primate origins and evolution. Princeton University Press, Princeton, Nova Jersey, EUA.

Mittermeier, R. A., Rylands, A. B., CoimbraFilho, A. F. \& Fonseca, G. A. B. 1988. Systematics: species and subspecies-an update. In: Mittermeier, R. A., Rylands, A. B., Coimbra-Filho, A. F. \& Fonseca, G. A. B. (eds.) Ecology and behaviour of Neotropical Primates. Academia Brasileira de Ciência, Rio de Janeiro, Brasil.

Moura, A. C. A. \& Lee, P. 2004. Capuchin stone tool use in Caatinga dry forest. Science, 306, 1909-1909.

Oliveira, M. M. \& Langguth, A. 2006. Rediscovery of Marcgrave's Capuchin Monkey and Designation of a Neotype for Simia flavia Schreber, 1774 (Primates, Cebidae). Boletim do Museu Nacional, 523, 116.

Oppenheimer, J. R. 1968. Behavior and ecology of the white-faced monkey, Cebus capucinus, on Barro Colorado Island, Canal Zone. University Microfilms International, Ann Arbor, Michigan, EUA.

Ortiz, R. E., Ortiz, A. C., Gajardo, G., Zepeda, A. J., Parraguez, V. H., Ortiz, M. E. \& Croxatto, H. B. 2005. Cytologic, hormonal, and ultrasonographic correlates of the menstrual cycle of the New World monkey Cebus apella. American Journal of Primatology, 66, 233244.

Overmier, J. B. \& Seligman, M. E. 1967. Effects of inescapable shock upon subsequent escape and avoidance responding. Journal of Comparative and Physiological Psychology, 63, 28-33.

Parr, L. A., Matheson, M. D., Bernstein, I. S. \& De Wall, F. B. M. 1997. Grooming down the hierarchy: allogrooming in captive brown capuchin monkeys, Cebus apella. Animal Behaviour, 54, 361-367.

Perry, S. E., Barrett, B. J. \& Godoye, I. 2017. Older, sociable capuchins (Cebus capucinus) invent more social behaviors, but younger monkeys innovate more in other contexts. Proceedins of the National Academy of Sciences of the United States of America, 114, 7806-7813. 
Social relationships in wild white-faced capuchin monkeys, Cebus capucinus. Dissertação (Doutorado), University of Michigan, EUA.

Pinha, P. S. 2007. Interações sociais em grupos de macacos-prego (Cebus libidinosus) no Parque Nacional de Brasília. Dissertação (Mestrado), Programa de Pós Graduação em Ecologia, Universidade de Brasília, Brasília. 67f.

Pissinatti, L., Tortelly, R., Porto, M., Burity, C. H. F. \& Pissinatti, A. 2008. Morfologia macroscópica do aparelho reprodutor feminino de Leontopithecus cativos (Lesson, 1840) Primates-Callitrichidae. Arquivo Brasileiro de Medicina Veterinária e Zootecnia, 60, 14721475.

Popilskis, S. J., Lee, D. R. \& Elmore, D. B. 2008. Anesthesia and Analgesia in Nonhuman Primates. In: Fish, R. E., Brown, M. J., Danneman, P. J. \& Karas, A. Z. Anesthesia and Analgesia in Laboratory Animals. American College of Laboratory Animal Medicine Series, Melbourne, Australia.

Raubenheimer, D. 2010. Foraging models. In: Breed, M. D. \& Moore, J. Encyclopedia of animal behavior. Academic Press, Oxford, Inglaterra.

Resende, B. D. 2004. Ontogenia de comportamentos manipulativos em um grupo de macacos-prego (Cebus apella) em situação de semiliberdade. Tese (Doutorado), Instituto de Psicologia, Universidade de São Paulo, São Paulo. 126f.

Robinson, J. G. \& Janson, C. H. 1987. Capuchins, squirrel monkeys, and atelines: socioecological convergence with old world primates. In: Smuts, B. B., Chenney, D. L., Seylarth, R. M., Wrangham, R. W. \& Struhsaker, T. T. (eds.) Primates societie. The University of Chicago Press, Chicago, EUA.

Rocha, V.J. 1992. Desenvolvimento de um método de manejo envolvendo um grupo de macacos-prego (Cebus apella) em condições semi-selvagem no Horto Florestal da UEL, Londrina-PR. Monografia (Zooecologia), Centro de Ciências Biológicas, Universidade Estadual de Londrina, Londrina.

Rocha, V. J., Reis, N. R. \& Sekiama, M. L. 1998. Uso de ferramentas por Cebus apella (Linnaeus)(Primates, Cebidae) para obtenção de larvas de coleóptera que parasitam sementes de Syagrus romanzoffianum (Cham.) Glassm.(Arecaceae). Revista Brasileira de Zoologia, 15, 945-950.
Rodrigues, R. C. 2010. Ciclo reprodutivo de macacos-prego (Cebus libidinosus) em cativeiro: Aspectos comportamentais e hormonais. Dissertação (Mestrado), Programa de pós-graduação em Ciências da saúde, Universidade de Brasília, Brasília. 70 f.

Rodrigues, R. C. 2016. Efeitos do tratamento prolongado de testosterona associado ao uso de algestona acetofenida e enantato de estradiol sobre o ciclo menstrual de fêmeas cativas de Sapajus libidinosus (macaco-prego). Tese (Doutorado), Programa de Pós Graduação em Biologia Animal, Universidade de Brasília, Brasília, 2016. 147f.

Rose, L. M. 1998. Behavioral ecology of whitefaced capuchins (Cebus capucinus) in Costa Rica. Dissertação (Doutorado), Washington University, EUA.

Rowe, N. 1996. Pictorial guide to the living primates. Pogonias Press, USA.

Rylands, A. B. \& Mittermeier, R. A. 2009. The diversity of the New World primates (Platyrrhini): an annotated taxonomy. In: IUCN (ed.) South American primates. Springer.

Sacramento, T. S. 2014. Influência da disponibilidade de alimentos sobre os comportamentos de um grupo de Sapajus libidinosus e análise das interações e conflitos entre humanos e macacos-prego no Parque Nacional de Brasília, DF. Dissertação (Mestrado), Programa de Pós-Graduação em Biologia Animal, Universidade de Brasília, Brasília. 79f.

Saletti, P. G. 2010. Avaliação da capacidade de discriminação do sinal cromático da "pele sexual" de Cebus libidinosus. Dissertação (Mestrado), Programa de Pós-Graduação em Ciências da Saúde, Universidade de Brasília, Brasília. 51f.

Santos, L. P. C. 2015. Parâmetros nutricionais da dieta de duas populações de macacos-prego: Sapajus libidinosus em ecótono cerrado/cattinga e Sapajus nigritus em área de Mata Atlântica. Tese (Doutorado), Programa de pós-graduação em Psicologia, Universidade de São Paulo, São Paulo. 1090 f.

Saunders, C. D. 1987. Grooming quality in relation to tick density and reciprocity between partners. American Journal of Primatology, 12,369 . 
Schino, G. 2001. Grooming, competition and social rank among female primates: a metaanalysis. Animal Behaviour, 62, 265-271.

Schino, G., Scucchi, S., Maestripieri, D. \& Turillazzi, P. G. 1988. Allogrooming as a tension-reduction mechanism: a behavioral approach. American Journal of Primatology, $16,43-50$.

Silva Junior, J. S. 2002. Taxonomy of capuchin monkeys, Cebus ERXLEBEN, 1777. Neotropical Primates, 2, 29.

Silva, E. D. R. 2008. Escolha de alvos coespecíficos na observação do uso de ferramentas por macacos-prego (Cebus libidinosus) selvagens. Dissertação (Mestrado), Programa de Pós-Graduação em Psicologia, Universidade de São Paulo, São Paulo. 87 p.

Silva, N. L. T. 2012. Acuidade visual e matriz extracelular no córtex visual primário: Alterações associadas à privação monocular precoce e ao enriquecimento ambiental. Dissertação (Mestrado), Pós-graduação em neurociências e biologia celular, Universidade Federal do Pará, Pará. 65f.

Silva, T. C. F. 2010. Estudo da variação na pelagem e da distribuição geográfica em Cebus flavius (Schreber, 1774) e Cebus libidinosus (Spix, 1823) do Nordeste do Brasil. Programa de Pós-graduação em Ciências Biológicas. Universidade Federal da Paraíba, João Pessoa.

Simpson, S. J. \& Raubenheimer, D. 2012. The nature of nutrition: a unifying framework from animal adaptation to human obesity. Princeton University Press, Princeton, Nova Jersey, EUA.

Snowdon, C. T. 1999. O significado da pesquisa em comportamento animal. Estudos de Psicologia, 4, 365-373.

Spironelo, W. R. 1991. Importância dos Frutos de Palmeiras (Palmae) na Dieta de um Grupo de Cebus apella (Cebidae, Primates) na Amazônia Central, p. 285-296. In: Rylands, A. B. \& Bernardes, A. T. (Eds). A Primatologia no Brasil. Fundação Biodiversitas, Paraíba, João Pessoa.

Stammbach, E. \& Kummer, H. 1982. Individual contributions to a dyadic interaction: an analysis of baboon grooming. Animal Behaviour, 30, 964-971.
Sussman, R. W., Garber, P. A. \& Cheverud, J. M. 2005. Importance of cooperation and affiliation in the evolution of primate sociality. American Journal of Physical Anthropology, 128, 84-97.

Terborgh, J. 1983. Five New World Primates. A study in comparative ecology. Princeton University Press, Princeton, Nova Jersey, EUA.

Torres de Assumpção, C. 2014. Resultados preliminares de reavaliação das raças do macaco-prego Cebus apella (Primates: Cebidae). Revista Nordestina de Biologia, 6, 15-28.

Torres de Assumpção, C. 1983. An ecological study of the primates of southeastern Brazil, with a reappraisal of Cebus apella races. Dissertação (PhD), University od Edinburg, Great-Britain.

Veras, M. M., Miglino, M. A. \& Silva, Z. 2007. Morfologia do aparelho reprodutor em fêmeas de bugio marrom (Alouatta guariba clamitans). Brazilian Journal of Veterinary Research and Animal Science, 44, 12-17.

Villar, D. N. A. 2006. Population census of Cebus libidinosus in the Altamiro de Moura Pacheco State Park, an area of Cerrado in Goiania (GO). XII Congresso Brasileiro de Primatologia, Livro de Resumos do XII Congresso Brasileiro de Primatologia.

Visalberghi, E. 1988. Responsiveness to objects in two social groups of tufted capuchin monkeys (Cebus apella). American Journal of Primatology, 15, 349-360.

Waga, I. C. 2008. Memória relacional espacial e não-espacial associada ao forrageamento em macacos-prego (Cebus spp.) mantidos em cativeiro. Dissertação (Mestrado), Programa de pós-graduação em Biologia Animal, Universidade de Brasília, Brasília. 67f.

\section{Article History: \\ Received 19 August 2017 \\ Accepted 9 September 2017 \\ Available on line 20 November 2017}

License information: This is an open-access article distributed under the terms of the Creative Commons Attribution License 4.0, which permits unrestricted use, distribution, and reproduction in any medium, provided the original work is properly cited. 